\title{
Random and channeling stopping powers of He and Li ions in $\mathrm{Si}$
}

\author{
G. de M. Azevedo, M. Behar, J. F. Dias, P. L. Grande, and D. L. da Silva \\ Instituto de Física, Universidade Federal do Rio Grande do Sul, Caixa Postal 15051, CEP 91501-970, Porto Alegre (RS), Brazil \\ G. Schiwietz \\ Hahn-Meitner-Institüt, Bereich Festkörperphysick, Glienicker Strasse 100, 14109 Berlin, Germany
}

(Received 26 October 2000; revised manuscript received 11 May 2001; published 16 January 2002)

\begin{abstract}
In this work we have measured the electronic stopping powers of ${ }^{4} \mathrm{He}$ and ${ }^{7} \mathrm{Li}$ ions for channeling and random directions in $\mathrm{Si}$ as a function of the incident ion energy. The channeling $(\langle 100\rangle$ for $\mathrm{Li},\langle 110\rangle$ and $\langle 111\rangle$ for $\mathrm{Li}$ and $\mathrm{He}$ ) and the random ( $\mathrm{Li}$ only) measurements cover a wide energy range between $200 \mathrm{keV}$ and 9 $\mathrm{MeV}$. The Rutherford backscattering technique together with different multilayer targets has been employed in the present experiments. The results are compared to calculations carried out in the framework of the unitary convolution approximation, which takes into account the impact-parameter-dependent energy loss for each projectile charge state, with further refinements including screening effects in close collisions between the electrons of the target and the screened projectile.
\end{abstract}

DOI: 10.1103/PhysRevB.65.075203 PACS number(s): 61.85.+p, 34.50.Bw, 34.50.Fa, 79.20.Rf

\section{INTRODUCTION}

The energy loss of light projectiles is an important issue not only because of its direct application in ion beam analysis and modifications of materials but also to improve the understanding of fundamental ion-solid interactions. For instance, for a precise ion beam analysis of the elemental depth distribution in near-surface layers, the stopping power of the respective ion-target combination must be known sufficiently well. ${ }^{1}$ The random stopping power of $\mathrm{H}$ and $\mathrm{He}$ ions in virtually any target is well documented in the literature. ${ }^{2}$ That is particularly true for light ions in amorphous $\mathrm{Si}$. On the other hand, there is a general lack of information concerning the slowing down of medium to heavy ions in amorphous Si. These data are important for two main reasons: from the fundamental point of view, in order to test current theoretical calculations for the electronic energy loss and, from the practical one, stopping power data of heavier ions are needed in analytic techniques like elastic recoil detection analysis (ERDA) or heavy-ion Rutherford backscattering (HIRBS) in order to perform precise energy to depth conversions.

Concerning the channeling stopping powers, the situation is quite different. There is a large amount of data related to $\mathrm{H}$ impinging along the Si major directions. For $\mathrm{He}$ in $\mathrm{Si}$, however, the information is scarce despite the fact that He beams are often used under channeling conditions in Si samples. In the pioneer work of Eisen et al., ${ }^{3}$ measurements of He in the $\langle 110\rangle$ and $\langle 111\rangle$ Si directions were performed by using the transmission geometry technique. Later on, dos Santos et al. ${ }^{4}$ and more recently Lulli et $a .^{5}$ and Yamamoto et al. ${ }^{6}$ reported $\mathrm{He}$ stopping data for the $\langle 100\rangle \mathrm{Si}$ channel. Concerning heavier ions, dos Santos et al. ${ }^{7}$ reported channeling results for $\mathrm{B}$ while the Heidelberg and the Bologna groups did the same for N. ${ }^{8,9}$ More recently, in the course of the present work, Jiang et al. ${ }^{10}$ reported results of axial channeling and random stopping powers for several medium-heavy ions in Si for a restricted energy range interval.

In the present work we have measured the electronic stopping power as a function of the incident ion energy for He at channeling and $\mathrm{Li}$ at channeling and random directions in $\mathrm{Si}$ crystals. The He measurements were done for the $\langle 110\rangle$ and $\langle 111\rangle$ axial directions, whereas the $\mathrm{Li}$ channeling experiments were performed for the three main directions. For these measurements we have used the Rutherford backscattering (RBS) channeling technique in combination with a SIMOX target. In addition, the $\mathrm{Li}$ values for random directions in $\mathrm{Si}$ were determined using the RBS technique together with a multilayer marker system. The advantage of the present experimental arrangement is that it circumvents the use of thin self-supported films as they are needed in transmission measurements. Moreover, the present technique allows stopping power measurements down to low energies. Consequently, we were able to measure in a wide energy range, between $200 \mathrm{keV}$ and $9 \mathrm{MeV}$.

The present results are compared with first-principles calculations that take into account the ion-flux distribution and the impact-parameter-dependent energy loss for each projectile charge state according to the unitary convolution approximation (UCA). ${ }^{11}$ This model was further extended here to include screening effects in close collisions because the $\mathrm{He}$ and Li ions carry bound electrons in the present energy range.

Finally, it must be pointed out that a partial account of the present theoretical-experimental results has been recently published as a Letter. ${ }^{12}$

\section{EXPERIMENTAL AND ANALYSIS PROCEDURES}

\section{A. Channeling stopping power}

We have used SIMOX-type samples consisting of $2000 \AA \mathrm{Si}\langle 100\rangle$ crystal layers on top of $4000 \AA \mathrm{SiO}_{2}$ buried layers, produced in $\langle 100\rangle \mathrm{Si}$ wafers. The samples were prepared at IBM (T.J. Watson Research Center). For each experiment, the sample was cleaned and etched to remove the native oxide film on the surface using a $10 \% \mathrm{HF}$ acid right before the RBS measurements. A summary of the present experimental conditions is given in Table I. 
TABLE I. The energy interval investigated in this work according to the charge state and the direction of helium and lithium ions.

\begin{tabular}{lccc}
\hline \hline Ion & Incident charge state & Direction & Energy range $(\mathrm{MeV})$ \\
\hline${ }^{4} \mathrm{He}$ & $1+$ & $\langle 110\rangle$ & $0.28-4.0$ \\
${ }^{4} \mathrm{He}$ & $2+$ & $\langle 110\rangle$ & $5.0-6.0$ \\
${ }^{4} \mathrm{He}$ & $1+$ & $\langle 111\rangle$ & $0.38-4.0$ \\
${ }^{4} \mathrm{He}$ & $2+$ & $\langle 111\rangle$ & 5.0 \\
\hline${ }^{7} \mathrm{Li}$ & $1+$ & $\langle 100\rangle$ & $0.47-4.0$ \\
${ }^{7} \mathrm{Li}$ & $2+$ & $\langle 100\rangle$ & $5.0-7.0$ \\
${ }^{7} \mathrm{Li}$ & $3+$ & $\langle 100\rangle$ & 8.0 \\
${ }^{7} \mathrm{Li}$ & $1+$ & $\langle 110\rangle$ & $0.55-4.0$ \\
${ }^{7} \mathrm{Li}$ & $2+$ & $\langle 110\rangle$ & 5.0 \\
${ }^{7} \mathrm{Li}$ & $1+$ & $\langle 111\rangle$ & $0.64-4.0$ \\
\hline${ }^{7} \mathrm{Li}$ & $1+$ & Random & $0.24-4.0$ \\
${ }^{7} \mathrm{Li}$ & $2+$ & Random & $4.3-7.0$ \\
${ }^{7} \mathrm{Li}$ & $3+$ & Random & $7.0-8.3$ \\
\hline \hline
\end{tabular}

The energy-loss measurements were carried out using the ion beam (with energy $E_{0}$ ) impinging on the sample at channeling and random directions. Based on a recent study ${ }^{13}$ and taking as references the $\langle 100\rangle$ axis $(\Psi=0)$ and the $\{100\}$ plane $(\phi=0)$, we have chosen as a random direction the one defined by $\Psi=6^{\circ}$ and $\phi=15^{\circ}$. The backscattered He or Li particles were detected by Si surface barrier detectors located at $170^{\circ}$ with respect to the incident beam. The overall resolution of the detection system was about $12 \mathrm{keV}$ for $\mathrm{He}$ and $20 \mathrm{keV}$ for Li. Typical RBS random and channeling spectra obtained for $1.2 \mathrm{MeV}{ }^{4} \mathrm{He}$ in the $\mathrm{Si}\langle 110\rangle$ direction and 2 $\mathrm{MeV}{ }^{7} \mathrm{Li}$ in the $\mathrm{Si}\langle 111\rangle$ direction are shown in Fig. 1. In this figure, $E_{c}$ and $E_{r}$ are the detected energies of the backscattered particles at the $\mathrm{Si} / \mathrm{SiO}_{2}$ interface for the channeled and random incidence, respectively. These energies were determined by fitting the channeling and random spectra with an algorithm ${ }^{14}$ which includes, in addition to the error function (accounting for particle straggling and detector resolution), the energy dependence of the Rutherford cross section. Finally, in the framework of the mean energy approximation, it can be shown ${ }^{4}$ that the channeling specific energy loss along the projectile incoming path (before the backscattering) is a function of the energies $E_{0}, E_{c}$, and $E_{r}$ of the specific energy loss in the random direction and of geometrical factors. For the helium case, we have used the random energy loss data of Niemann et al., ${ }^{15}$ while for the lithium case we used the experimental values obtained in this work.

At this point, it is important to stress that the channeling stopping power depends on the target thickness because of the ion flux distribution and projectile charge state. For very thin crystals, the ion flux distribution is nearly uniform and the projectile charge state is equal to the incident one. After less than $2000 \AA$, equilibrated ion flux distributions ${ }^{4}$ and projectile charge states ${ }^{16}$ are achieved. Then, assuming that the effect of the preequilibrium projectile charge state is of minor importance (because an incident charge state close to the equilibrium value can be chosen), the channeling stopping power will be nearly identical to the random one. On

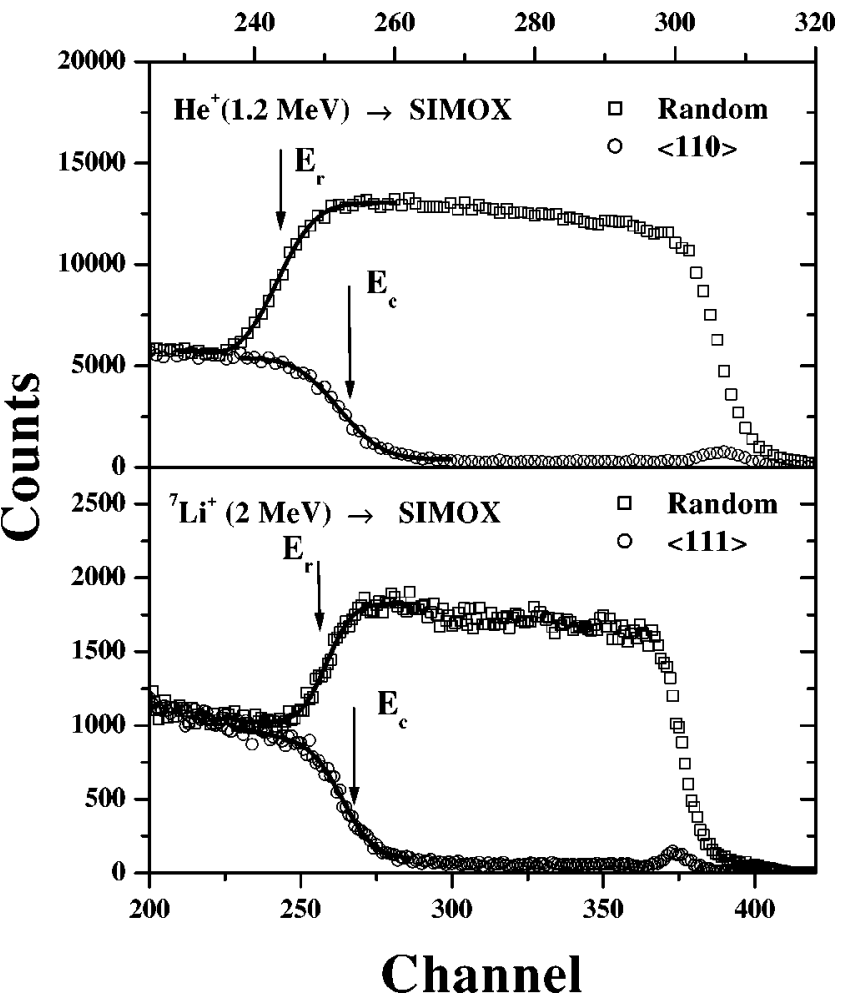

FIG. 1. RBS spectra for $1.2 \mathrm{MeV} \mathrm{He}{ }^{+}$and $2 \mathrm{MeV}^{7} \mathrm{Li}^{+}$for the $\langle 110\rangle$ and $\langle 111\rangle$ directions in $\mathrm{Si}$, respectively. In this figure, $E_{c}$ and $E_{r}$ stand for the detected energies of the backscattered particles from the $\mathrm{Si} / \mathrm{SiO}_{2}$ interface for channeling and random conditions, respectively. Solid lines represent the results of the fitting procedure (Ref. 14) used to determine the corresponding energy loss.

the other hand, if the crystal is too thick, the channeling stopping power also may approach the random one due to dechanneling at crystal defects, thermal vibrations, and electronic multiple scattering. The present experimental conditions, using SIMOX targets of $2000 \AA$, is thick enough to prevent a preequilibrium ion flux distribution and thin enough to avoid enhanced dechanneling effects.

\section{B. Random stopping power}

For these experiments we have used two types of markers. The first one, suitable for the higher-energy measurements, is a sandwich system composed of eight $\mathrm{Sb}$ layers deposited alternatively on amorphous $\mathrm{Si}$ ones $\left(\mathrm{Si} / \mathrm{Sb}_{8}\right)$ by using the molecular beam epitaxy (MBE) technique. The whole system was grown on top of a $\mathrm{SiO}_{2}$ film built on a $\mathrm{Si}\langle 100\rangle$ substrate. The thickness of the $\mathrm{Sb}$ markers is about $20 \AA$ according to measurements using transmission electron microscopy (TEM). The average surface roughness of $10 \AA$, as determined with an atomic force microscope, is negligible for our purposes.

The second system, suitable for the low-energy measurements, consists of three different Au markers implanted in a $\mathrm{Si}\langle 100\rangle$ substrate $\left(\mathrm{Si} / \mathrm{Au}_{1}\right)$. In this case, we have first amorphized the $\mathrm{Si}$ wafers using an $\mathrm{Ar}$ beam $(\Phi=2$ $\times 10^{14}$ atoms $/ \mathrm{cm}^{2}, E=300 \mathrm{keV}$ ). Afterwards, Au ions were implanted at $30 \mathrm{keV}, 50 \mathrm{keV}$, or $100 \mathrm{keV}$ at fluences of 


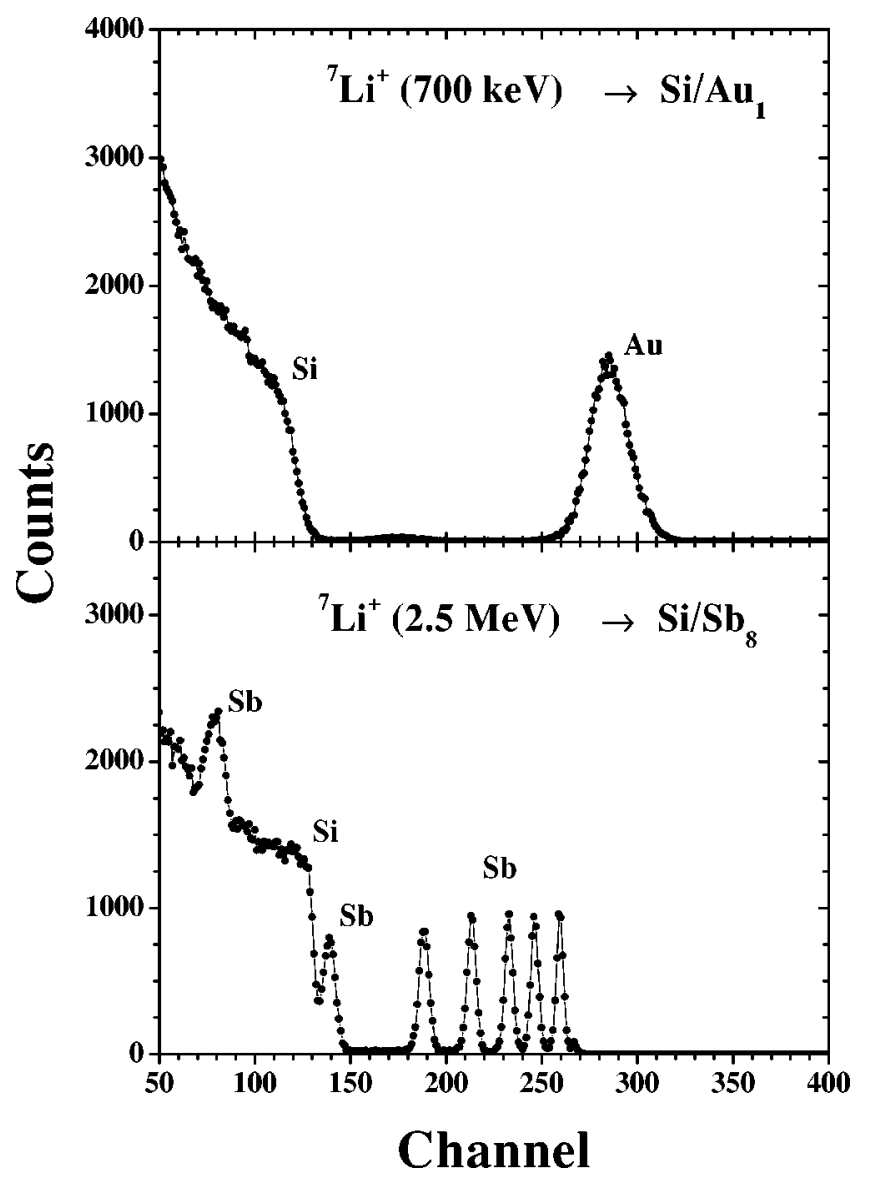

FIG. 2. RBS spectra obtained for ${ }^{7} \mathrm{Li}^{+}$impinging on a $\mathrm{Si} / \mathrm{Au}_{1}$ single marker and on a $\mathrm{Si} / \mathrm{Sb}_{8}$ multilayer marker.

about $\Phi=10^{15}$ atoms $/ \mathrm{cm}^{2}$. These fluences are too low to modify the stopping power of the material, but high enough to provide good sensitivity for the backscattering experiments.

The determination of the position of the markers in the $\mathrm{Si} / \mathrm{Sb}_{8}$ multilayer and in the $\mathrm{Si} / \mathrm{Au}_{1}$ targets were done using 1-3 MeV He beams provided by the $3 \mathrm{MV}$ Tandetron of the Institute of Physics of Porto Alegre. The RBS measurements were performed at two geometries: one with the beam normal to the sample (normal geometry), while the other one with the sample at four different angles between $20^{\circ}$ and $60^{\circ}$ with respect to the beam (tilted geometry).

The RBS spectra taken with a Li beam corresponding to the multilayer and marker systems are shown in Fig. 2. As discussed in some detail in Ref. 7, for a given beam of energy $E_{0}$, at least two independent measurements at different geometries are necessary in order to determine $d E / d x$ at both incoming and backscattered paths simultaneously in the framework of the mean energy approximation. In this work, each quoted $(d E / d x)$ is a mean value obtained from at least five independent measurements at different geometries.

\section{THEORETICAL PROCEDURE}

\section{A. Energy loss of channeled ions}

Under channeling conditions, the energy loss due to the Si inner-shell electrons is strongly suppressed, since the ion flux distribution along a $\mathrm{Si}$ major axial direction has a peak in the middle of the channel (flux peaking). Hence, the mean energy lost by the projectile after passing a certain thickness $X$ is given by the general formula

$$
\Delta E=\frac{\int_{A} d^{2} \rho \int_{0}^{X} d x \frac{d E(\vec{\rho})}{d x} \Phi(\vec{\rho}, x)}{\int_{A} d^{2} \rho \int_{0}^{X} d x \Phi(\vec{\rho}, x)}
$$

where $A$ is the transversal area of the Si axial channel, $\vec{\rho}$ is the position relative to the center of the channel, and $\Phi(\vec{\rho}, x)$ is the ion flux distribution at the distance $\vec{\rho}$ and channeling direction $x$. The energy loss per traversed distance, $(d E / d x)(\vec{\rho})$, may be divided into two contributions:

$$
\frac{d E}{d x}(\vec{\rho})=\left(\frac{d E}{d x}(\vec{\rho})\right)_{\text {direct }}+\left(\frac{d E}{d x}(\vec{\rho})\right)_{\text {e-loss }} .
$$

The first contribution corresponds to the energy loss involving the Si electrons (target ionization and excitation and electron capture), while the second one is due to energy loss from electrons originally bound to the projectile (projectile ionization and excitation). Both contributions can be obtained from the impact-parameter-dependent energy loss in binary collisions between the projectile in a charge state $q$ with a neutral $\mathrm{Si}$ atom as

$$
\left(\frac{d E}{d x}(\vec{\rho})\right)_{\text {direct }}=\sum_{q} f_{q} \sum_{i} \frac{Q_{\text {direct }}^{(q)}\left(b_{i}\right)}{d},
$$

where $d$ is the interatomic distance along the axial channel and the sum over $i$ has to be performed according to the projected atomic structure given by Fig. 3. The trajectory averaged electron density of atomic Si orbitals deviates by only $2.5 \%$ from its solid-state value. Solid-state effects due to the Si valence electrons - namely, the partially compensating collective screening and plasmon excitation-have implicitly been accounted for by normalizing the mean energy transfers of all shells to yield the Bethe value as extracted from experimental solid-state data. Within first-order perturbation theory, the results of our method (without Bloch terms) agree with full band-structure calculations for protons in $\mathrm{Si}$ (Ref. 17) to within about 3\% for the total stopping power as well as for the channeling energy loss. Finally, since the projectiles may be found in different charge states $q$, with fraction given by $f_{q}$, the average over the projectile charge state has to be performed. The He and Li charge-state distributions were taken from experiments performed under channeling conditions. ${ }^{10,18}$ For He ions with energies larger than $1.1 \mathrm{MeV}$, extrapolated values have been used.

In order to obtain the ion flux distribution, we have used the string-potential model. ${ }^{19}$ Consequently, only the transverse motion has to be determined. For Si axial channeling, Ziegler-Biersack-Littmark (ZBL) string potentials were added to calculate the potential energy as a function of ion transversal position $\vec{\rho}$ according to Fig. 3 for each Si main axis. We have solved Newton's equations of motion numeri- 


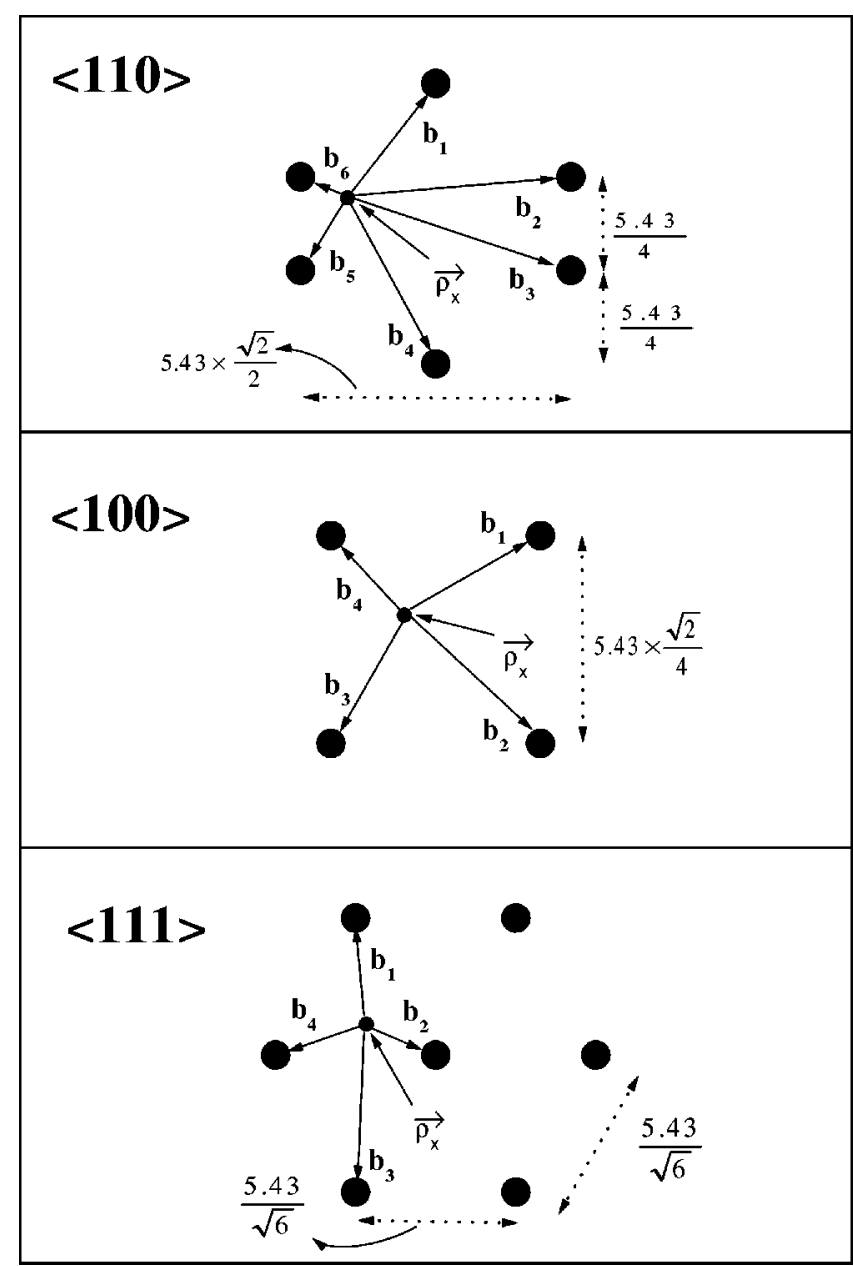

FIG. 3. Coordinate space of the $\langle 110\rangle,\langle 100\rangle$, and $\langle 111\rangle$ channels of the Si crystal. Parameters relevant to the calculations are shown in the pictures. Numerical values are quoted in angstroms. The drawings involve different scales. See text for further information.

cally for an ensemble of ions impinging on the channel at normal incidence. Thus, the two-dimensional transverse motion and the ion-flux distribution as a function of the penetration depth were calculated. Thermal vibrations have been included in the simulations by convoluting the string potential with a Gaussian distribution that describes transversal displacements of the $\mathrm{Si}$ atoms. Here, we have considered the root mean square of these displacements as $\sigma=0.083 \AA$ according to the Debye temperature from Refs. 13 and 20. Other methods such as Monte Carlo calculations ${ }^{21}$ yield similar flux distributions.

\section{B. Impact-parameter-dependent energy loss $Q(b)$}

The energy loss $Q(b)$ as a function of the impact parameter $b$ in a single collision plays a very important role in the determination of the stopping power under channeling conditions. In fact, the reduction of the stopping power for channeled ions is due to a significant suppression of collisions with small impact parameters. Full calculations of the impact-parameter-dependent energy loss using first-order perturbation theory have been performed only recently, ${ }^{22}$ fol- lowed later by the first coupled-channel calculations. ${ }^{23,24}$ These calculations are time consuming and results are available only for a few selected collision systems. Therefore, the computer-simulation community has preferred to use semiempirical formulas $^{25}$ as well as other simplified ${ }^{26}$ or even oversimplified $^{27}$ models in order to deal with inelastic collisions. Here, we shall adopt the more advanced unitary convolution approximation (UCA) as described below.

\section{Unitary convolution approximation}

A detailed description of the UCA model for bare ions may be found elsewhere. ${ }^{11}$ Here we present only a short outline of the method and its extension for screened projectiles. The electronic energy loss is defined by

$$
Q(b)=\sum_{f}\left|a_{f}(\vec{b})\right|^{2}\left(\epsilon_{f}-\epsilon_{0}\right)
$$

which involves a sum over all final target states with energies $\epsilon_{f}$ and the corresponding transition amplitudes $a_{f}$, for each impact parameter. Even in a first-order Born approximation for the amplitudes $a_{f}$, direct calculation of $Q(b)$ from Eq. (4) demands a computational effort that precludes its use in a computer-simulation code. In particular, the sum over all final states in Eq. (4) involves very high angular momentum values and converges very slowly for screened projectiles at high energies.

In the framework of first-order perturbation theory, a simple formula for $Q(b)$ has been proposed recently ${ }^{28}$ involving a convolution of the target electronic density with an analytical kernel. This kernel represents the interaction strength between the projectile and a quasifree electron. An extension of this model for projectiles carrying bound electrons was described in Ref. 29. This formula is able to reproduce first-order Born results ${ }^{28}$ with very high accuracy for all impact parameters. The convolution model was further extended to take into account higher-order effects arising from the probability conservation in close collisions in the framework of Bloch approximation. It was shown that this extended model, called the UCA, provides reliable energy loss values for highly charged swift projectiles. The extension of this model for projectiles carrying bound electrons will be described in what follows by considering a screened potential for the interaction between the projectile ion and the target electrons. This potential contains not only the Coulomb part due to the nuclear charge of the projectile, but also the static potential produced by the projectile electrons that screen the projectile-nuclear charge.

The UCA formula is extended here to take screened projectiles into account by considering the same type of product ansatz as in Ref. 11,

$$
Q(b)=\int d^{2} r_{T} \mathcal{K}\left(\vec{b}-\vec{r}_{T}\right) \int d x \rho\left(\vec{r}_{T}, x\right),
$$




$$
\mathcal{K}\left(b_{e}\right)=\frac{2 Z_{p}^{2}}{v_{p}^{2} b_{e}^{2}} h\left[2 v_{p} b_{e} / \eta\left(Z_{e f f}\right)\right] \sum_{i} f_{i} g\left(\frac{\omega_{i} b_{e}}{v_{p}}\right),
$$

where $Z_{p}$ and $v_{p}$ are the projectile nuclear charge and velocity, respectively, and $b_{e}$ is the relative impact parameter (between the projectile and the target electron). The electronic density $\rho(\vec{r})$ (Ref. 30) is integrated along the ion path. The kernel $\mathcal{K}$ yields a smooth interpolation between small and large impact parameters for which there exist analytical formulas for $Q(b) .^{28}$ The first two terms in Eq. (6) describe violent binary collisions between the screened projectile and the target electron, and the last term accounts for the screening effect by strongly reducing the long ranged dipole transitions. The first integral $\int d^{2} r_{T} \ldots$ in Eq. (5) describes a convolution with the initial electron density. According to Ref. 29, the functions $g(x)$ and $h(x)$ read

$$
\begin{aligned}
g(x)= & {\left[x^{2}+\left(\alpha b_{e}\right)^{2}\right] K_{1}^{2}\left[\sqrt{x^{2}+\left(\alpha b_{e}\right)^{2}}\right] } \\
& +x^{2} K_{0}^{2}\left[\sqrt{x^{2}+\left(\alpha b_{e}\right)^{2}}\right]
\end{aligned}
$$

and

$$
h(x)=\frac{x^{2}}{2} \int_{0}^{1} d y y K_{0}\left(x y^{2}\right) J_{0}\left(x y \sqrt{1-y^{2}}\right) .
$$

In the above equations, $K_{0}$ and $K_{1}$ are the modified Bessel functions, $\omega_{i}$ are the transition energies $\left(\omega_{i} \equiv \epsilon_{i}-\epsilon_{0}\right), f_{i}$ are the well-known dipole-oscillator strengths $\left[f_{i}\right.$ $\left.=2|\langle i|z| 0\rangle|^{2}\left(\boldsymbol{\epsilon}_{i}-\boldsymbol{\epsilon}_{0}\right)\right]$, which fulfill the sum rule $\Sigma_{i} f_{i}=1.31$ The parameter $\alpha$ is the reciprocal of the screening length of a Bohr-like (or Yukawa-type) projectile screening potential. Expressions for the function $g$ for other screening potentials may be found in Ref. 29. The scaling factor $\eta$ is defined in Ref. 11 as

$$
\begin{aligned}
\eta & =\exp [\operatorname{Re} \psi(1+i \gamma)-\psi(1)] \\
& =\exp \left[\gamma^{2} \sum_{l=1}^{\infty} \frac{1}{l\left(l^{2}+\gamma^{2}\right)}\right]
\end{aligned}
$$

with $\gamma=Z_{e f f} / v_{p}$. In this work, the projectile screening is taken into account for close collisions by using an impactparameter-dependent effective charge $Z_{e f f}$. The values of $Z_{\text {eff }}$ are obtained from the screened projectile potential as

$$
Z_{e f f}\left(b_{e}\right)=Z_{p}-n_{e} \Phi\left(b_{e}\right),
$$

where $\Phi(r)$ is the projectile-screening function. In this way, the bound projectile electrons will reduce the interaction between the projectile and target electrons. It is important to point out that the projectile electrons screen the projectile nucleus as spectators of the target excitation; i.e., the projectile electrons remain in the ground state when the target is excited. Thus, we also neglect the so-called antiscreening effect, where the electron-electron interaction between the bound projectile electrons and the target electrons results in an enhancement of the ionization and excitation cross sections at intermediate to high energies. ${ }^{32-34}$ Nevertheless, at

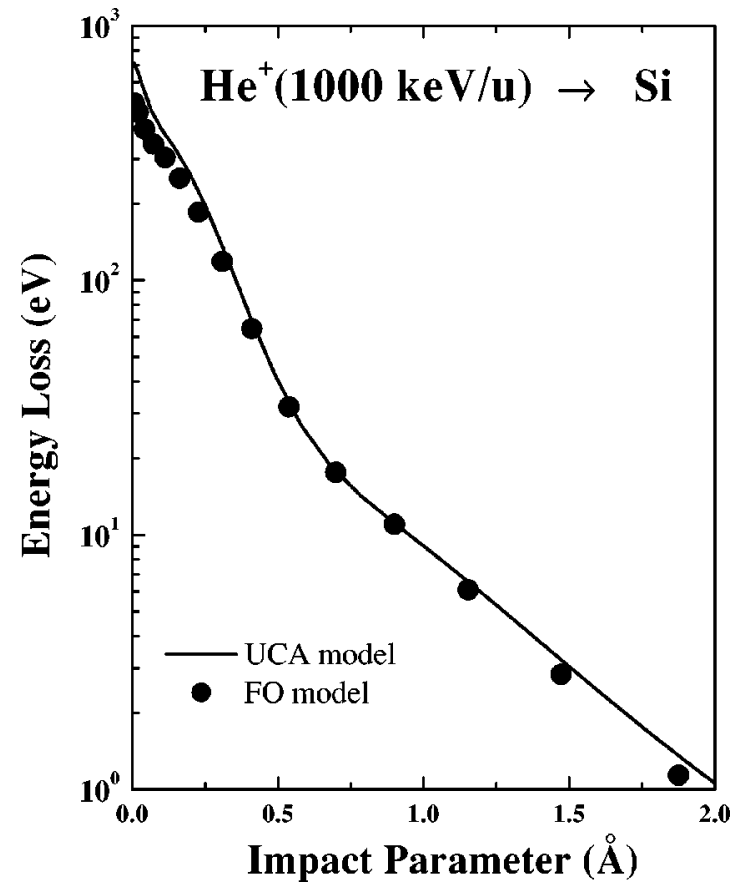

FIG. 4. Energy loss as a function of the impact parameter $b$ for $1000 \mathrm{keV} /$ nucleon He ions impinging on a Si target. Solid line: UCA model. Solid circles: first-order perturbation theory. See text for further information.

these energies, the projectile-charge state is generally about the projectile atomic number and, consequently, the antiscreening effect is of minor importance for energy loss calculations.

A comparison of the present model with other calculations is shown in Figs. 4 and 5 for the energy loss of $1 \mathrm{MeV} /$ nucleon $\mathrm{He}^{+}$ions colliding with $\mathrm{Si}$ atoms. Two energy loss processes are relevant for this collision system: namely, the direct ionization and excitation of $\mathrm{Si}$ by $\mathrm{He}^{+}$projectiles (Fig. 4) as well as the projectile ionization and excitation by the $\mathrm{Si}$ target (Fig. 5). The first process can be well described in first-order perturbation theory ${ }^{31}$ (FO) for screened He ions impinging on $\mathrm{Si}$. These calculations (FO) are represented in Fig. 4 by solid circles and the ones corresponding to the UCA model are depicted by a solid line. For both models, the appropriate screening potential is the single- $\zeta$ potential as described in Ref. 29 (with $Z_{p}^{e f f}=2$ ). For the target parameters, the electronic density of the wave functions in the FO model for each subshell was taken into account. For each subshell, however, only a single oscillator strength and a mean transition energy was considered in the UCA model, according to the analysis performed in Ref. 29. As can be observed, the agreement of the UCA with FO calculations is very good. However, for small impact parameters $(b \ll 1)$, deviations are found, being more significant at lower projectile energies. The reason for that is an overestimated innershell contribution to the calculations. That reflects the limitation of the UCA model which is strictly valid for projectile speeds exceeding the mean orbital electron velocity.

The second energy loss process is due to the projectile ionization and excitation by the $\mathrm{Si}$ atoms. These atoms can 


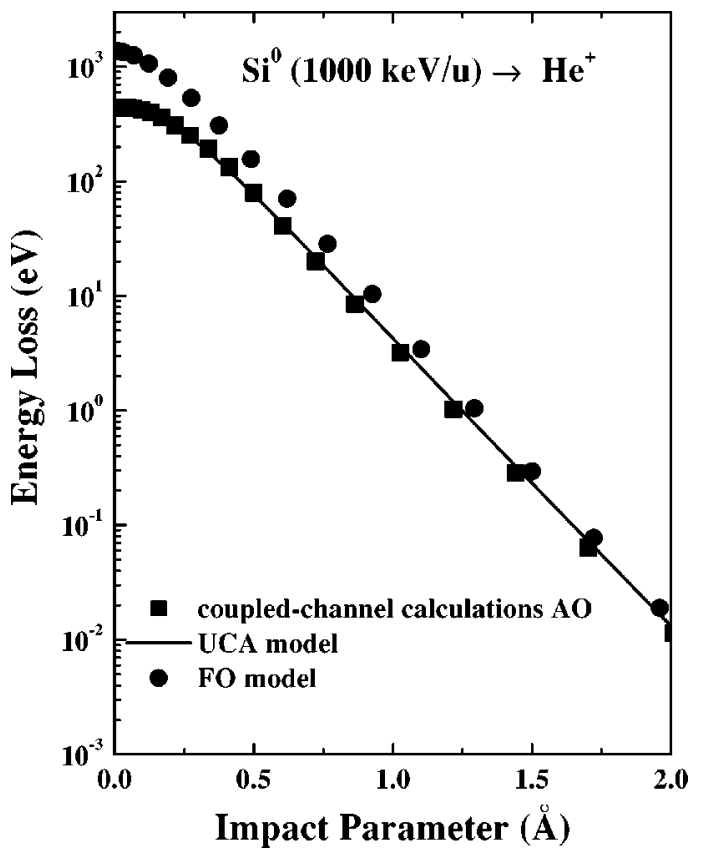

FIG. 5. Energy loss as a function of the impact parameter $b$ for $1000 \mathrm{keV} /$ nucleon $\mathrm{Si}^{0}$ ions impinging on a $\mathrm{He}^{+}$target. Solid line: UCA model. Solid squares: coupled-channel calculations. Solid circles: first-order perturbation theory. See text for further information.

be seen as "projectiles" in the reference frame of the $\mathrm{He}^{+}$ ions. Since this energy loss mechanism involves a strong perturbation due to the large nuclear charge of $\mathrm{Si}$, we have to compare the present model to nonperturbative calculations as well. Therefore, we compare the present model with the coupled-channel calculations that provides the most reliable values for the projectile-electron loss process. The coupledchannel method is based on the numerical solution of the time-dependent Schrödinger equation in the framework of the independent particle model. This method ${ }^{23}$ describes the energy loss very accurately ${ }^{24}$ and is used here as a benchmark for comparison with our present energy-loss model. According to Fig. 5, there is an excellent agreement between the present UCA model (solid line) and the coupled-channel calculation (solid squares). Here the same projectile screening function was used in both calculations, namely the general screening function as given in Ref. 29. It is important to note that first-order calculations lead to strong deviations from the accurate coupled-channel results as indicated in Fig. 5.

\section{RESULTS AND DISCUSSIONS}

\section{A. Channeling stopping power}

According to the procedure outlined in Sec. II A, we have determined the energy loss of ${ }^{4} \mathrm{He}$ ions along $\mathrm{Si}\langle 110\rangle$ and $\langle 111\rangle$ Si directions using the random stopping values of Niemann $e t a l .{ }^{15}$ (see Table I for the respective energy ranges). The results are presented in Fig. 6 together with the channeling data by Lulli et al. ${ }^{5}$ and Eisen $e t a l .,{ }^{3}$ as well as with the random stopping values by Niemann et al. ${ }^{15}$ Each experi-

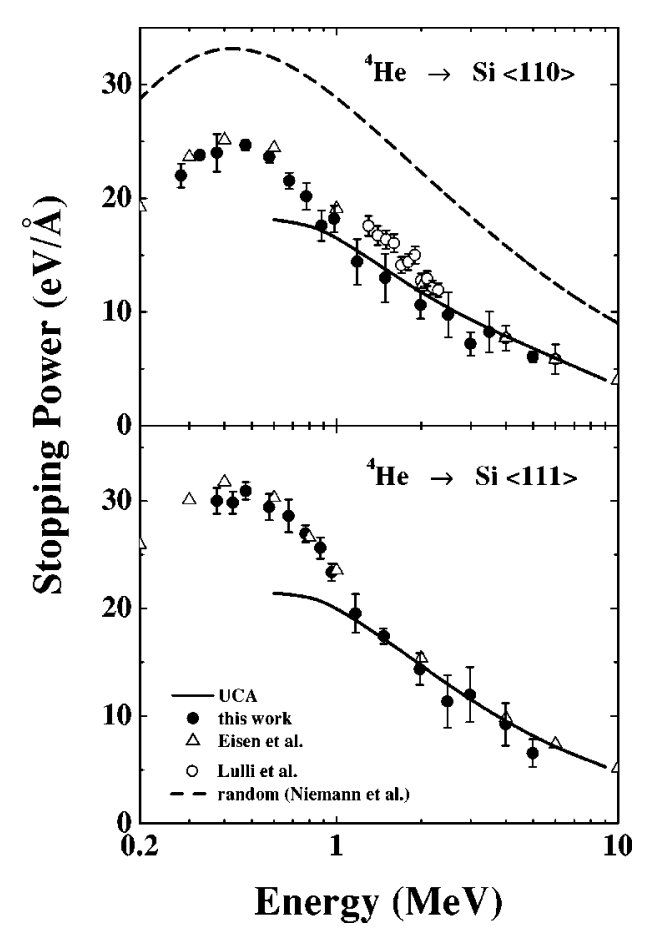

FIG. 6. Energy loss of $\mathrm{He}$ ions as a function of the ion energy for the $\langle 110\rangle$ and $\langle 111\rangle$ directions in Si. Solid circles: this work. Solid line: UCA model. Open circles: Lulli et al. (Ref. 5). Open triangles: Eisen et al. (Ref. 3). Dashed line: random stopping power by Niemann et al. (Ref. 15).

mental point obtained in this work corresponds to an average of at least five individual measurements. The quoted errors (not only in Fig. 6 but also in Figs. 7 and 8) were estimated from the statistical dispersion of the individual measurements. An inspection of Fig. 6 indicates that the stopping powers corresponding to the $\langle 110\rangle$ channel are about $10-$ $30 \%$ lower than the ones corresponding to the $\langle 111\rangle$ channel. This feature is a direct consequence of the fact that the $\langle 110\rangle$ channel is much wider than the $\langle 111\rangle$ (see Fig. 3). In addition, it can be observed that both specific energy loss curves have a maximum in the same energy region, around $400 \mathrm{keV}$. The channeling stopping power decreases faster with increasing energy than the random one. This general behavior reflects the role of the valence and inner-shell electrons. Indeed, for increasing projectile energies, the contribution of the inner-shell electrons to the random stopping power increases whereas the contribution from these electrons are almost completely suppressed under axial channeling conditions. Furthermore the maximum channeling to random stopping ratio ( $\alpha$ ratio) attained for the $\langle 111\rangle$ and $\langle 110\rangle$ directions are $\approx 0.9$ and 0.7 , respectively. Bearing in mind that the random and channeling stopping powers originate mostly from the interaction of the ion with the valence electrons of the target, the difference observed in the maximum $\alpha$ ratio values can be explained in terms of the distribution of valence electrons across the channels. In the case of the $\langle 111\rangle$ channel, these electrons are almost homogeneously distributed across this channel. On the other hand, the $\langle 110\rangle$ being the widest channel, the reduced valence-electron density is reflected by a maximum $\alpha$ ratio significantly smaller than 1 . 


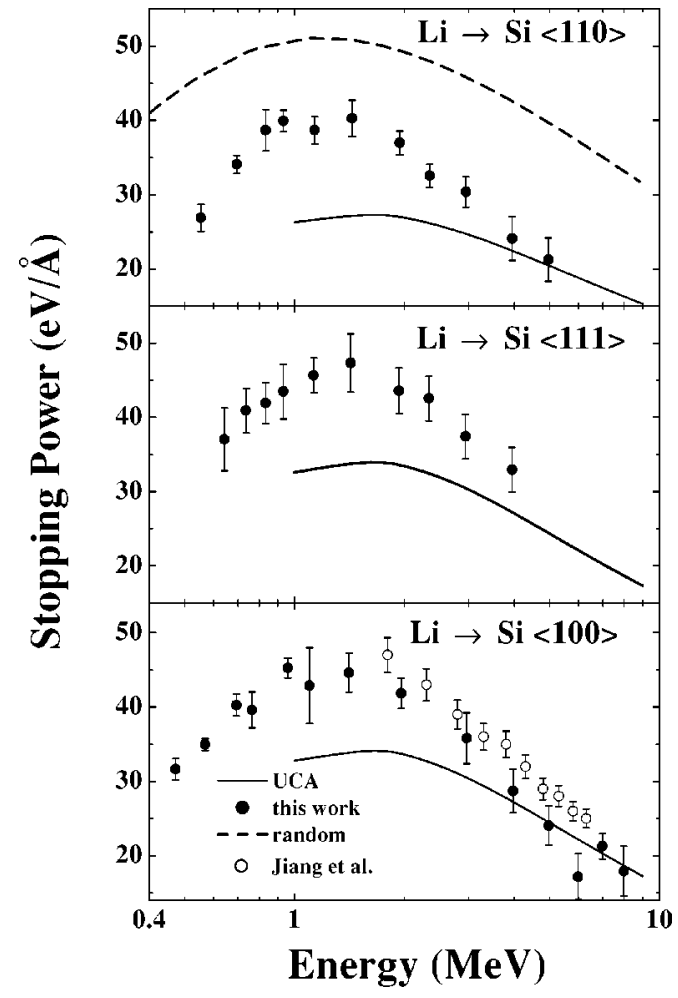

FIG. 7. Energy loss of Li ions as a function of the ion energy for the $\langle 100\rangle,\langle 110\rangle$, and $\langle 111\rangle$ directions in Si. Solid circles: this work. Solid lines: UCA model. Open circles: Jiang et al. (Ref. 10). Dashed line: random stopping power calculated with Eq. (A1) and the values quoted in Table II.

A comparison of our results (solid symbols) with previous experimental channeling data (open symbols) (Refs. 3 and 5) is also depicted in Fig. 6. As can be observed, up to $1 \mathrm{MeV}$ there is a quite good agreement among the different sets of data. However, for intermediate energies (1-3 MeV), our results are systematically lower (of the order of 10-15\%) than those reported by Lulli et al. ${ }^{5}$ (open circles). Nevertheless, the difference is still within the experimental errors. It should be stressed the previous channeling data have been measured using thick targets (about $10000 \AA$ ). This fact may introduce a slight overestimation of the channeling energy loss due to dechanneling at crystal defects and electronic multiple scattering.

As shown in Fig. 6, the improved UCA calculations agree fairly well with data for high energies. Conversely, for energies lower than $1.5 \mathrm{MeV}$, the calculations underestimate the measured stopping power values. It has been shown ${ }^{12}$ that this difference comes from polarization effects which are not included in the present calculations.

In Fig. 7 the ${ }^{7} \mathrm{Li}\langle 100\rangle,\langle 110\rangle$, and $\langle 111\rangle$ channeling stopping powers are displayed. It should be mentioned that the part of the data for the $\langle 100\rangle$ channel was published previously, ${ }^{35}$ and they are included here only for the sake of completeness. As discussed before, in order to obtain the channeling data, we have to use the random stopping power as an input for the data analysis. In this case, we have used the random stopping powers measured in this work and discussed in the next subsection. The energy range covered for

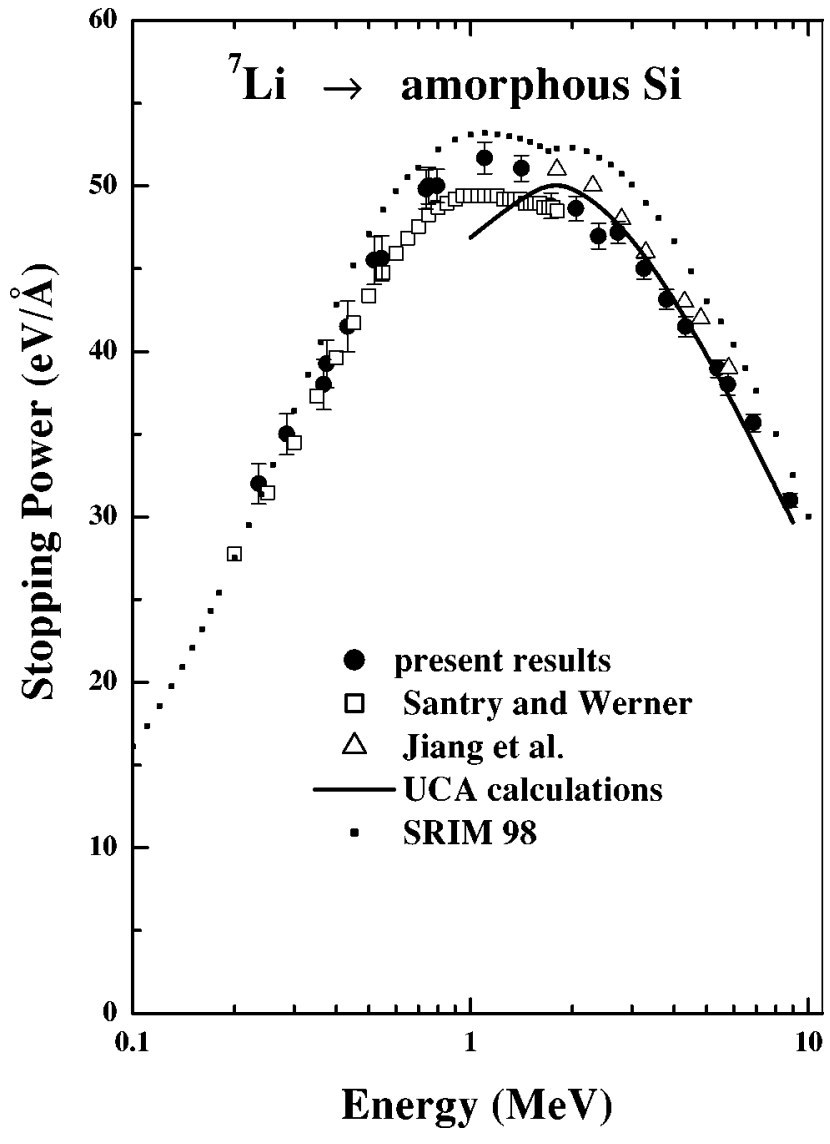

FIG. 8. Energy loss of Li ions as a function of the ion energy in amorphous Si. Solid circles: this work. Solid line: UCA model. Dotted line: SRIM 98 (Ref. 37). Open squares: Santry et al. (Ref. 36). Open triangles: Jiang et al. (Ref. 10).

each axial channel was different (see Table I for details). By the time the present work was under way, Jiang et al. ${ }^{10}$ have published Li channeling data for the $\langle 100\rangle \mathrm{Si}$ direction in a restricted energy range using the ion transmission technique. These data are shown as open circles in Fig. 7. The experimental points of Jiang et al. ${ }^{10}$ are systematically higher than the present ones, the difference being of the order of $10 \%$. As in the case of $\mathrm{He}$, a slight overestimation of the channeling energy loss is expected for the transmission geometry. This is due to the fact that effects such as dechanneling at crystal defects and electronic multiple scattering are more pronounced, since much thicker targets have to be employed for this technique.

As in the case of He, the UCA calculations underestimate the experimental $\mathrm{Li}$ results for low to intermediate energies (up to 4-5 MeV). However, the disagreement is much larger for Li than for He ions. Again, the difference is related to high-order effects which are not accounted for in the UCA approximation. In this case, the polarization effect, which depends on $Z_{p}^{3}$, seems to be responsible for the observed differences. ${ }^{12}$

\section{B. Li random stopping power}

In Fig. 8, the Li random stopping powers obtained in the present experiment are shown as solid circles. The data is 
TABLE II. Parameters obtained by fitting Eq. (A1) to the data discussed in this work. See text for further information.

\begin{tabular}{|c|c|c|c|c|c|c|c|c|}
\hline Ion & $\begin{array}{l}\text { Energy } \\
(\mathrm{MeV})\end{array}$ & Direction & $\alpha_{0}$ & $\alpha_{1}$ & $\alpha_{2}$ & $\alpha_{3}$ & $\alpha_{4}$ & $\beta$ \\
\hline${ }^{4} \mathrm{He}$ & $0.38-4.98$ & $\langle 111\rangle$ & $7.178 \times 10^{-2}$ & $-4.446 \times 10^{-2}$ & $-7.886 \times 10^{-2}$ & $6.648 \times 10^{-2}$ & $4.861 \times 10^{-2}$ & 1.557 \\
\hline${ }^{4} \mathrm{He}$ & $0.28-5.99$ & $\langle 110\rangle$ & $8.179 \times 10^{-2}$ & $-4.989 \times 10^{-2}$ & $-8.941 \times 10^{-2}$ & $7.522 \times 10^{-2}$ & $1.095 \times 10^{-1}$ & 6.418 \\
\hline${ }^{7} \mathrm{Li}$ & $0.47-7.98$ & $\langle 100\rangle$ & $1.216 \times 10^{-1}$ & $-8.130 \times 10^{-3}$ & $-6.576 \times 10^{-2}$ & $1.305 \times 10^{-2}$ & $5.317 \times 10^{-2}$ & 192.86 \\
\hline${ }^{7} \mathrm{Li}$ & $0.64-3.95$ & $\langle 111\rangle$ & $1.106 \times 10^{-1}$ & $-2.79 \times 10^{-2}$ & $-7.381 \times 10^{-2}$ & $5.317 \times 10^{-2}$ & $2.09 \times 10^{-2}$ & 31.42 \\
\hline${ }^{7} \mathrm{Li}$ & $0.55-4.96$ & $\langle 110\rangle$ & $1.129 \times 10^{-1}$ & $-4.119 \times 10^{-2}$ & $-9.218 \times 10^{-2}$ & $4.922 \times 10^{-2}$ & $2.54 \times 10^{-2}$ & 5.734 \\
\hline${ }^{7} \mathrm{Li}$ & $0.24-8.83$ & Random & $6.092 \times 10^{-2}$ & $-1.051 \times 10^{-1}$ & $7.331 \times 10^{-2}$ & $2.157 \times 10^{-2}$ & $6.57 \times 10^{-3}$ & 15.7 \\
\hline
\end{tabular}

well fitted (not shown here) by an expression similar to that one proposed by the Kalbitzer group. ${ }^{15}$ The fitting formula can be found in the Appendix, and the fitted parameters are shown in Table II. In addition to our data, we show the data previously published by Santry and $\mathrm{Werner}^{36}$ (open squares) and by Jiang et al. ${ }^{10}$ (open triangles). These data sets cover a different but yet complementary energy range. An inspection of this figure shows that the present results are in quite good agreement with both previous measurements and the difference does not exceed the $8 \%$ level. In the same figure, we show the semiempirical interpolations by Ziegler, Biersack, and Littmark ${ }^{37}$ obtained from the computer code SRIM 98. As can be observed, this procedure reproduces fairly well the experimental results. At intermediate to high energies SRIM 98 tends to overestimate the present results, the difference being of the order of $8 \%$.

It is interesting to compare the UCA calculations with the random stopping of $\mathrm{Li}$ in amorphous $\mathrm{Si}$. As can be observed in Fig. 8, good agreement is obtained between the present results and the UCA calculations down to $2.0 \mathrm{MeV}$. This feature is at variance with what was observed for the channeling experiments, where the UCA results clearly underestimate the experimental values. However, the abovementioned agreement is accidental. In fact, the UCA model is valid only for projectile speeds exceeding the mean electron orbital velocities. It is expected that this limitation will result in overestimated stopping powers due to the Si innershell electrons. Thus, the neglected higher-order effects such as the polarization (Barkas effect) and the overestimated stopping power for Si inner-shell electrons are compensating each other.

\section{CONCLUSIONS}

In the present work we have measured the channeling stopping power of ${ }^{4} \mathrm{He}$ and ${ }^{7} \mathrm{Li}$ as well as the random stopping power of ${ }^{7} \mathrm{Li}$ in $\mathrm{Si}$ targets. For the channeling measurements we have used the RBS technique associated with a SIMOX target. This method allowed us to perform the measurements in a wide energy range without changing the experimental conditions.

The He stopping powers were investigated along the $\mathrm{Si}$ $\langle 110\rangle$ and $\langle 111\rangle$ directions in a $0.3-6 \mathrm{MeV}$ energy range. We have found that the stopping powers measured along the $\langle 110\rangle$ directions are systematically $10-30 \%$ lower than those obtained along the $\langle 111\rangle$ direction because of the geometry of these channels. A comparison with previous experimental data performed using transmission technique shows a good agreement up to $1 \mathrm{MeV}$. For higher energies, the previous experimental data are systematically higher than the present ones with a difference of about $8-10 \%$. This might be related to the thick targets used in previous transmission technique experiments.

The Li channeling stopping data obtained in a $0.5-8 \mathrm{MeV}$ energy interval shows the same features as the He results. However, a significantly reduced energy loss for the widest Si channel $\langle 110\rangle$ is observed. Comparison with the previous experimental data (obtained for the $\mathrm{Si}\langle 100\rangle$ channel using the transmission geometry) indicates a similar discrepancy as for He projectiles.

The Li random stopping data were obtained in the 0.2-9.0 $\mathrm{MeV}$ energy range by using the RBS technique and different marker systems. Our data are in very good agreement with those obtained by Santry and Werner ${ }^{36}$ and by Jiang et al. ${ }^{10}$ A comparison between the present results with the semiempirical calculations by ZBL indicates uncertanties of less than $10 \%$ from the ZBL predictions.

Concerning the theoretical calculations based on the UCA approximation for the impact-parameter-dependent energy loss, we have observed good agreement with the channeling experimental data for energies down to $2 \mathrm{MeV}$ for $\mathrm{He}$ and at the highest energies for Li. It has been shown ${ }^{12}$ that the difference between the experimental data and UCA calculations is due to polarization effects (Barkas). On the other hand, the random experimental data are well reproduced by UCA calculations for a much wider energy range. However, for the lower-energy range this agreement should be taken as accidental. This comes from the fact that while the UCA calculations may overestimate the energy loss at close collisions with inner-shell electrons, it underestimates the energy loss at distant collisions. Therefore, for random incidence where both distant and close collisions contribute equally, a compensation of neglected effects results in good agreement between theory and experiment.

\section{ACKNOWLEDGMENTS}

This work was partially supported by the Brazilian agencies Conselho Nacional de Desenvolvimento Científico e 
Tecnológico (CNPq) and CAPES-DAAD (BrazilianGerman) international cooperation program (PROBRAL).

\section{APPENDIX}

The present $\mathrm{He}$ and Li random and channeling data can be fitted with the following semiempirical expression worked out from the published results by Niemann et al.: ${ }^{15}$

$$
\frac{d E}{d x}=\frac{E^{1 / 2} \ln (2.71828+\beta E)}{\alpha_{0}+\alpha_{1} E^{1 / 4}+\alpha_{2} E^{1 / 2}+\alpha_{3} E+\alpha_{4} E^{3 / 2}},
$$

where $\alpha_{0}, \alpha_{1}, \alpha_{3}, \alpha_{4}$, and $\beta$ are fitting parameters and $E$ is the ion energy. Table II gives the ions ( $\mathrm{He}$ or $\mathrm{Li}$ ), the energy range for which the fit is valid, the direction, and the parameters obtained for each case. In the above expression, the stopping power is given in $\mathrm{eV} / \AA$ for ion energies quoted in $\mathrm{MeV}$.
${ }^{1}$ W.K Chu, J.W. Mayer, and M.A. Nicolet, Backscattering Spectrometry (Academic, New York, 1978).

${ }^{2}$ H.H. Andersen and J.F. Ziegler, Hydrogen Stopping Powers and Ranges in All Elements (Pergamon, New York, 1977), Vol. III; J.F. Ziegler, Helium Stopping Powers and Ranges in All Elements (Pergamon, New York, 1977), Vol. IV.

${ }^{3}$ F.H. Eisen, G.J. Clark, J. Bottiger, and J.M. Poate, Radiat. Eff. 13, 93 (1972).

${ }^{4}$ J.H.R. dos Santos, P.L. Grande, M. Behar, H. Boudinov, and G. Schiwietz, Phys. Rev. B 55, 4332 (1997).

${ }^{5}$ G. Lulli, E. Albertazzi, M. Bianconi, G.G. Bentini, R. Nipoti, and R. Lotti, Nucl. Instrum. Methods Phys. Res. B 170, 1 (2000).

${ }^{6}$ Y. Yamamoto, A. Ikeda, T. Yoneda, K. Kajiyama, and Y. Kido, Nucl. Instrum. Methods Phys. Res. B 153, 10 (1999).

${ }^{7}$ J.H.R. dos Santos, M. Behar, P.L. Grande, and H. Boudinov, Phys. Rev. B 55, 13651 (1997).

${ }^{8}$ D. Niemann, G. Konac, and S. Kalbitzer, Nucl. Instrum. Methods Phys. Res. B 118, 11 (1996).

${ }^{9}$ G.G. Bentini, M. Bianconi, R. Nipoti, F. Malaguti, and E. Verondini, Nucl. Instrum. Methods Phys. Res. B 53, 1 (1991).

${ }^{10}$ W. Jiang, R. Grötzschel, W. Pilz, B. Schmidt, and W. Möller, Phys. Rev. B 59, 226 (1999); 60, 714 (1999).

${ }^{11}$ G. Schiwietz and P.L. Grande, Nucl. Instrum. Methods Phys. Res. B 153, 1 (1999).

${ }^{12}$ G. De M. Azevedo, P.L. Grande, M. Behar, J.F. Dias, and G. Schiwietz, Phys. Rev. Lett. 86, 1482 (2001).

${ }^{13}$ A. Dygo, W.N. Lennard, and I.V. Mitchell, Nucl. Instrum. Methods Phys. Res. B 84, 23 (1994).

${ }^{14}$ J.H.R. dos Santos, P.L. Grande, H. Boudinov, M. Behar, R. Stoll, Chr. Klatt, and S. Kalbitzer, Nucl. Instrum. Methods Phys. Res. B 106, 51 (1994).

${ }^{15}$ D. Niemann, G. Konac, and S. Kalbitzer, Nucl. Instrum. Methods Phys. Res. B 118, 11 (1996).

${ }^{16}$ G. de M. Azevedo, J.R.A. Kaschny, J.F. Dias, P.L. Grande, M. Behar, Ch. Klatt, and S. Kalbitzer, Nucl. Instrum. Methods Phys. Res. B 148, 168 (1999).

${ }^{17}$ J.M. Pitarke and I. Campillo, Nucl. Instrum. Methods Phys. Res. B 164-165, 147 (2000).

${ }^{18}$ R.J. Petty and G. Dearnaley, Phys. Lett. A 50, 273 (1974).
${ }^{19}$ J. Lindhard, Mat. Fys. Medd. K. Dan. Vidensk. Selsk. 34, 14 (1965).

${ }^{20}$ G. de M. Azevedo, J.C. Martini, M. Behar, and P.L. Grande, Nucl. Instrum. Methods Phys. Res. B 149, 301 (1999).

${ }^{21}$ J.H. Barret, Phys. Rev. B 3, 1527 (1971).

${ }^{22}$ N.M. Kabachnik, V.N. Kondratev, and O.V. Chumanova, Phys. Status Solidi B 145, 103 (1988).

${ }^{23}$ G. Schiwietz, Phys. Rev. A 42, 296 (1990).

${ }^{24}$ G. Schiwietz and P.L. Grande, Nucl. Instrum. Methods Phys. Res. B 69, 10 (1992); P.L. Grande and G. Schiwietz, Phys. Rev. A 47, 1119 (1993); Nucl. Instrum. Methods Phys. Res. B 132, 264 (1997).

${ }^{25}$ O.S. Oen and M.T. Robinson, Nucl. Instrum. Methods 132, 647 (1976).

${ }^{26}$ O.B. Firsov, Zh. Éksp. Teor. Fiz. 36, 1517 (1959) [Sov. Phys. JETP 9, 1076 (1959)].

${ }^{27}$ J. Lindhard and A. Winther, K. Dan. Vidensk. Selsk. Mat. Fys. Medd. 34, 1 (1964).

${ }^{28}$ P.L. Grande and G. Schiwietz, Phys. Rev. A 58, 3796 (1998).

${ }^{29}$ G. de M. Azevedo, P.L. Grande, and G. Schiwietz, Nucl. Instrum. Methods Phys. Res. B 164, 203 (2000).

${ }^{30}$ F. Herman and S. Skillmann, Atomic Structure Calculations (Prentice-Hall, Englewood Cliffs, NJ, 1963).

${ }^{31}$ H.A. Bethe and R.W. Jackiw, Intermediate Quantum Mechanics 2nd ed. (Benjamin, New York, 1968).

${ }^{32}$ E.C. Montenegro, W.E. Meyerhof, and J.H. McGuire, Adv. At., Mol., Opt. Phys. 34, 249 (1994).

${ }^{33}$ N. Stolterfoht, Nucl. Instrum. Methods Phys. Res. B 53, 477 (1991).

${ }^{34}$ D.R. Bates and G.W. Griffing, Proc. Phys. Soc., London, Sect. A 68, 90 (1955).

${ }^{35}$ J.F. Dias, G. de M. Azevedo, M. Behar, P.L. Grande, Chr. Klatt, and S. Kalbitzer, Nucl. Instrum. Methods Phys. Res. B 148, 164 (1999).

${ }^{36}$ D.C. Santry and R.D. Werner, Nucl. Instrum. Methods Phys. Res. B 5, 449 (1984).

${ }^{37}$ J.F. Ziegler, J.P. Biersack, and U. Littmark, The Stopping and Range of Ions in Solids (Pergamon, New York, 1985). 